\title{
Mixed Norm Bergman-Morrey-type Spaces on the Unit Disc
}

\author{
A. N. Karapetyants ${ }^{1,2^{*}}$ and S. G. Samko ${ }^{3 * *}$ \\ ${ }^{1}$ Southern Federal University, Rostov-on-Don, Russia \\ ${ }^{2}$ Don State Technical University, Rostov-on-Don, Russia \\ ${ }^{3}$ Universidade do Algarve, Portugal \\ Received February 17, 2016
}

\begin{abstract}
We introduce and study the mixed-norm Bergman-Morrey space $\mathscr{A}^{q ; p, \lambda}(\mathbb{D})$, mixednorm Bergman-Morrey space of local type $\mathscr{A}_{\text {loc }}^{q ; p, \lambda}(\mathbb{D})$, and mixed-norm Bergman-Morrey space of complementary type ${ }^{\complement} \mathscr{A}^{q} ; p, \lambda(\mathbb{D})$ on the unit disk $\mathbb{D}$ in the complex plane $\mathbb{C}$. The mixed norm Lebesgue-Morrey space $\mathscr{L}^{q ; p, \lambda}(\mathbb{D})$ is defined by the requirement that the sequence of Morrey $L^{p, \lambda}(I)$-norms of the Fourier coefficients of a function $f$ belongs to $l^{q}(I=(0,1))$. Then, $\mathscr{A}^{q ; p, \lambda}(\mathbb{D})$ is defined as the subspace of analytic functions in $\mathscr{L}^{q ; p, \lambda}(\mathbb{D})$. Two other spaces $\mathscr{A}_{\text {loc }}^{q ; p, \lambda}(\mathbb{D})$ and $\complement_{\mathscr{A}^{q ; p, \lambda}}(\mathbb{D})$ are defined similarly by using the local Morrey $L_{\text {loc }}^{p, \lambda}(I)$-norm and the complementary Morrey ${ }^{\complement} L^{p, \lambda}(I)$-norm respectively. The introduced spaces inherit features of both Bergman and Morrey spaces and, therefore, we call them Bergman-Morrey-type spaces. We prove the boundedness of the Bergman projection and reveal some facts on equivalent description of these spaces.
\end{abstract}

DOI: $10.1134 / \mathrm{S} 000143461607004 \mathrm{X}$

Keywords: Bergman-Morrey-type space, mixed norm.

\section{INTRODUCTION}

Starting with the papers by Bergman [1] and Dzherbashyan [2], [3], the spaces of analytic functions which are $p$-integrable with respect to a sigma-finite measure on a connected open set in the complex plane $\mathbb{C}$ or in $\mathbb{C}^{n}$ have been intensively studied by a number of authors (see the books [4]-[10] and the references therein). The study of Toeplitz operators as well as algebras of Toeplitz operators acting in Bergman spaces served as an important objective for developing the entire theory of such spaces. The knowledge of the structural properties of these spaces, in particular, is very useful in studying Toeplitz-type operators on such spaces.

More recent advances in the theory of space of analytic functions are connected with the study of Bergman-type spaces and other spaces such as analytic Besov spaces, Q-spaces, Lipschitz, Bloch, BMOA, and their numerous analogues and generalizations. The variety of the definitions and approaches used to define and study such spaces allows to characterize them from different points of views, and still these spaces have a significant interplay among themselves, since they draw much from the classical theory of Bergman and even of Hardy spaces.

A major issue that passes through all the above is that the boundary behavior of a function from the space under consideration or the boundary behavior of the corresponding symbol of a Toeplitz operator is the most important point.

The introduction of a mixed norm is the natural generalization of the classical Bergman space, which, in particular, allows to distinguish between variables and, hence, to specify the boundary behavior of functions with more accuracy.

\footnotetext{
${ }^{*}$ E-mail: karapetyants@gmail.com

${ }^{* *}$ E-mail: ssamko@ualg.pt
} 
Several particular results on the mixed-norm Bergman spaces with integral mixed norm are known. These results are related to the boundedness of the Bergman projection and to some properties of Bergman spaces, such as duality, etc. Without claiming completeness, we refer the reader to the papers [11]-[15](also see the references therein).

Here we consider another generalization of the classical Bergman space, which is cardinally different from the above-mentioned approach. Instead of the angular integral norm, we use the $l^{q}$-norm of the sequence whose entries are Morrey-type norms of the Fourier coefficients of a function $f$ on $\mathbb{D}$. The introduced spaces inherit features of both Bergman and Morrey spaces and, therefore, we call them Bergman-Morrey-type spaces. General references on Morrey spaces are [16], [17]. We refer the reader to [18] for recent advances on real variable Morrey-type space theory. There are also some essential developments un the study of the classical operators of real analysis in general Morrey-type spaces; see [19]-[22] (see the references therein).

Note that, for $\lambda=0$, the Morrey-type spaces $L^{p, 0}(I), L_{\text {loc }}^{p, 0}(I)$, and $L^{p, 0}(I)$ are $L^{p}(I)$. The mixed-norm Bergman-type spaces in this case were considered in an even more general situation: in the paper [23], we used variable order Lebesgue space $L^{p(\cdot)}(I)$ to define the corresponding mixed-norm variable-order Bergman space over $\mathbb{D}$. So the paper [23] covers the results of the present paper for $\lambda=0$. The present paper may be considered as a natural continuation of the study of Bergman-type spaces with mixed-norm structure as in [23]. One of the underlying ideas of this study is an application of such results to the study of Toeplitz-type operators. We intent to consider such applications in another paper.

Note that the Morrey norm was already used for the analytic function space to measure boundary values of the Hardy $H^{2}(\mathbb{D})$ functions. The analytic Morrey spaces on the unit disc and upper half plane were introduced and studied in relation with Q-spaces in [24] (also see [25] for further development).

The article is organized as follows. In Sec. 2, we give the necessary definitions and notions on classical Bergman spaces, Bergman projection and Morrey-type spaces. In Sec. 3, we introduce the spaces

$$
\mathscr{L}^{q ; p, \lambda}(\mathbb{D}), \quad \mathscr{A}^{q ; p, \lambda}(\mathbb{D}), \quad \mathscr{L}_{\mathrm{loc}}^{q ; p, \lambda}(\mathbb{D}), \quad \mathscr{A}_{\mathrm{loc}}^{q ; p, \lambda}(\mathbb{D}), \quad \complement_{\mathscr{L}^{q ; p, \lambda}}(\mathbb{D}), \quad \complement_{\mathscr{A}}^{q ; p, \lambda}(\mathbb{D}) .
$$

In Sec. 4, we establish asymptotic estimates (asymptotic behavior) for the sequences of numbers

$$
\left\|r^{n}\right\|_{L^{p, \lambda}(I)}, \quad\left\|r^{n}\right\|_{L_{\text {loc }}^{p, \lambda}(I)}, \quad\left\|r^{n}\right\|_{L_{L^{p, \lambda}(I)}}, \quad n \in \mathbb{Z}_{+} \text {(nonnegative integers). }
$$

These estimates and asymptotics allow us to prove the boundedness of the Bergman projection as a projection from $\mathscr{L}^{q ; p, \lambda}(\mathbb{D})$ onto $\mathscr{A}^{q, p(\cdot)}(\mathbb{D})$, from $\mathscr{L}_{\text {loc }}^{q ; p, \lambda}(\mathbb{D})$ onto $\mathscr{A}_{\text {loc }}^{q ; p, \lambda}(\mathbb{D})$, and from ${ }^{\complement} \mathscr{L}^{q ; p, \lambda}(\mathbb{D})$ onto $\complement_{\mathscr{A}}^{q ; p, \lambda}(\mathbb{D})$ in Sec. 5. In Sec. 6, based on estimates from Sec. 4, we discuss equivalent norms in $\mathscr{A}^{q ; p, \lambda}(\mathbb{D}),{ }^{\complement} \mathscr{A}^{q ; p, \lambda}(\mathbb{D})$, and reveal connection of these spaces with the Hardy space $H^{2}(\mathbb{D})$, exploring the Hadamard's fractional derivatives as well.

\section{PRELIMINARIES AND DEFINITIONS}

\subsection{On Classical Hardy $H^{p}(\mathbb{D})$ and Bergman $\mathscr{A}^{p}(\mathbb{D})$ Spaces and Bergman Projection $B_{\mathbb{D}}$}

The references for the results and definitions stated here are [6]-[9]. For a function $\varphi$ on the unit disk $\mathbb{D}$ and for $0 \leq r<1$, we write

$$
\begin{array}{rlr}
\mathscr{M}_{p}(\varphi ; r)=\left\{\frac{1}{2 \pi} \int_{0}^{2 \pi}\left|\varphi\left(r, e^{i \theta}\right)\right|^{p} d \theta\right\}^{1 / p}, & 0<p<\infty, \\
\mathscr{M}_{p}(\varphi ; r)={\operatorname{ess}-\sup _{\theta \in[0,2 \pi)}\left|\varphi\left(r, e^{i \theta}\right)\right|,} \quad p=\infty .
\end{array}
$$

The class of analytic functions $f$ in $\mathbb{D}$ for which

$$
\|f\|_{H^{p}(\mathbb{D})} \equiv \lim _{r \rightarrow 1} \mathscr{M}_{p}(f ; r)<\infty, \quad 0<p \leq \infty
$$

is the Hardy class $H^{p}(\mathbb{D})$.

MATHEMATICAL NOTES Vol. 100 No. 12016 
Let $d A(z)$ stands for the area measure on $\mathbb{D}$ normalized so that the area of $\mathbb{D}$ is 1 . As usual, $\mathscr{A}^{p}(\mathbb{D})$ stands for the Bergman space of analytic functions $f$ in $\mathbb{D}$ that belong to $L^{p}(\mathbb{D})=L^{p}(\mathbb{D} ; d A(z))$. The corresponding Bergman projection $B_{\mathbb{D}}$, which is defined on $f \in L^{1}(\mathbb{D})$ as

$$
B_{\mathbb{D}} f(z)=\int_{\mathbb{D}} K(z, w) f(w) d A(w), \quad z \in \mathbb{D},
$$

is bounded from $L^{p}(\mathbb{D})$ onto $\mathscr{A}^{p}(\mathbb{D})$ for $1<p<\infty$.

$$
\begin{gathered}
\text { 2.2. Morrey Space } L^{p, \lambda}(I) \text {, Local Morrey Space } L_{\text {loc }}^{p, \lambda}(I) \text {, and Complementary Morrey Space }{ }^{\complement} L^{p, \lambda}(I) \\
\text { on } I=(0,1)
\end{gathered}
$$

There are numerous sources for Morrey space theory. We refer the reader to [18] for definitions and properties. Let $1 \leq p<\infty, \lambda \geq 0$. The Morrey space $L^{p, \lambda}(I)$ over the interval $I$ is defined as the set of measurable on $I$ functions $\varphi$ such that

$$
\sup _{r, r \pm h \in I, h>0} \frac{1}{(2 h)^{\lambda}} \int_{r-h}^{r+h}|\varphi(t)|^{p} 2 t d t<\infty .
$$

The local Morrey space $L_{\text {loc }}^{p, \lambda}(I)$ over the interval $I$ is defined as the set of measurable (on $I$ ) functions $\varphi$ such that

$$
\sup _{h \in I} \frac{1}{h^{\lambda}} \int_{1-h}^{1}|\varphi(t)|^{p} 2 t d t<\infty .
$$

Such local spaces are sometimes called central Morrey spaces. The complementary Morrey space ${ }^{\complement} L^{p, \lambda}(I)$ over the interval $I$ is defined as the set of measurable on $I$ functions $\varphi$ such that

$$
\sup _{h \in I} h^{\lambda} \int_{0}^{1-h}|\varphi(t)|^{p} 2 t d t<\infty .
$$

The $p$ th root from each expression above provides the corresponding norm.

The space $L^{p, \lambda}(I)$ is trivial for $\lambda>1$ and

$$
L^{p, 0}(I)=L_{\text {loc }}^{p, 0}(I)={ }^{\complement} L^{p, 0}(I)=L^{p}(I)
$$

and $L^{p, 1}(I)=L^{\infty}(I)$. The space ${ }^{\complement} L^{p, \lambda}(I)$ contains nonintegrable functions for $\lambda \geq p-1$.

Therefore, in what follows, we shall assume that $0 \leq \lambda<1$ for $L^{p, \lambda}(I), L_{\text {loc }}^{p, \lambda}(I)$, and $0 \leq \lambda<p-1$ for ${ }^{\complement} L^{p, \lambda}(I)$. Note that these space are not separable for $\lambda$ as specified above, unless $\lambda=0$.

\section{MIXED NORM BERGMAN-MORREY-TYPE SPACES

$$
\text { 3.1. Spaces } \mathscr{L}^{q ; p, \lambda}(\mathbb{D}), \mathscr{L}_{\mathrm{loc}}^{q ;, \lambda}(\mathbb{D}) \text {, and, }{ }^{\complement} \mathscr{L}^{q ; p, \lambda}(\mathbb{D})
$$
the rule

Given a function $f(z)=f\left(r, e^{i \alpha}\right)$ on $\mathbb{D}$ introduce the Fourier transform in angular variable defined by

$$
I \otimes \mathscr{F} f=\left\{f_{n}(r)\right\}_{n \in \mathbb{Z}}, \quad f_{n}(r)=\frac{1}{2 \pi} \int_{0}^{2 \pi} f\left(r, e^{i \alpha}\right) e^{-i n \alpha} d \alpha .
$$

Let further for each $n \in \mathbb{Z}$ the function $f_{n}=f_{n}(r)$ belong to the space $L^{p, \lambda}(I)$, where $1 \leq p<\infty$.

Introduce the mixed-norm space $\mathscr{L}^{q ; p, \lambda}(\mathbb{D}), 1 \leq q<\infty, 1 \leq p<\infty, 0 \leq \lambda<1$, as the space of measurable on $\mathbb{D}$ functions $f$ such that the Fourier coefficients $f_{n}(r)$ exist for almost all $r \in I$ and the norm

$$
\|f\|_{\mathscr{L} q ; p, \lambda(\mathbb{D})}=\left(\sum_{n \in \mathbb{Z}}\left\|f_{n}\right\|_{L^{p, \lambda}(I)}^{q}\right)^{1 / q}
$$

is finite. The mixed-norm spaces $\mathscr{L}_{\text {loc }}^{q ; p, \lambda}(\mathbb{D}), 1 \leq q<\infty, 1 \leq p<\infty, 0 \leq \lambda<1$, and ${ }^{\complement} \mathscr{L}^{q ; p, \lambda}(\mathbb{D})$, $1 \leq q<\infty, 1<p<\infty, 0 \leq \lambda<p-1$, are similarly defined with the $L^{p, \lambda}(I)$-norm in (3.1) replaced by the local Morrey $L_{\text {loc }}^{p, \lambda}(I)$-norm and the complementary Morrey ${ }^{\complement} L^{p, \lambda}(I)$-norm, respectively. 


\subsection{Bergman-Morrey-Type Spaces $\mathscr{A}^{q ; p, \lambda}(\mathbb{D}), \mathscr{A}_{\text {loc }}^{q ; p, \lambda}(\mathbb{D}),{ }^{\complement} \mathscr{A}^{q ; p, \lambda}(\mathbb{D})$}

We introduce the mixed-norm Bergman-Morrey space

$$
\mathscr{A}^{q ; p, \lambda}(\mathbb{D}), \quad 1 \leq q<\infty, \quad 1 \leq p<\infty, \quad 0 \leq \lambda<1,
$$

as the space of functions $\mathscr{L}^{q ; p, \lambda}(\mathbb{D})$ which are analytic in $\mathbb{D}$. Hence the norm of a function $f \in \mathscr{A}^{q ; p, \lambda}(\mathbb{D})$ is given by

$$
\|f\|_{\mathscr{A} q ; p, \lambda(\mathbb{D})}=\left(\sum_{n \in \mathbb{Z}_{+}}\left\|f_{n}\right\|_{L^{p, \lambda}(I)}^{q}\right)^{1 / q} .
$$

Similarly, the mixed-norm Bergman-Morrey space of local type

$$
\mathscr{A}_{\mathrm{loc}}^{q ; p, \lambda}(\mathbb{D}), \quad 1 \leq q<\infty, \quad 1 \leq p<\infty, \quad 0 \leq \lambda<1,
$$

and the mixed-norm Bergman-Morrey space of complementary type

$$
\complement_{\mathscr{A}^{q ; p, \lambda}}(\mathbb{D}), \quad 1 \leq q<\infty, \quad 1<p<\infty, \quad 0 \leq \lambda<p-1,
$$

are defined accordingly as the subspaces of $\mathscr{L}_{\text {loc }}^{q ; p, \lambda}(\mathbb{D})$ and ${ }^{\complement} \mathscr{L}^{q ; p, \lambda}(\mathbb{D})$ consisting of analytic functions in $\mathbb{D}$.

It will be convenient to use the following notation. Everywhere in the sequel, $X^{p, \lambda}(I)$ stands for one of the three spaces

(1) $X^{p, \lambda}(I)=L^{p, \lambda}(I), 1 \leq p<\infty, 0 \leq \lambda<1$,

(2) $X^{p, \lambda}(I)=L_{\text {loc }}^{p, \lambda}(I), 1 \leq p<\infty, 0 \leq \lambda<1$,

(3) $X^{p, \lambda}(I)={ }^{\complement} L^{p, \lambda}(I), 1<p<\infty, 0 \leq \lambda<p-1$.

Remark 1. By the definition of the spaces under consideration, the Fourier coefficients $f_{n}=f_{n}(r)$, $n \in \mathbb{Z}$, of a function $f$ in any of Bergman-Morrey-type spaces $\mathscr{A}^{q ; p, \lambda}(\mathbb{D}), \mathscr{A}_{\mathrm{loc}}^{q ; p, \lambda}(\mathbb{D})$, or ${ }^{\complement} \mathscr{A}^{q ; p, \lambda}(\mathbb{D})$ have the form

$$
f_{n}(r)= \begin{cases}a_{n}\left\|r^{n}\right\|_{X^{p, \lambda}(I)}^{-1} r^{n}, & n \in \mathbb{Z}_{+}, \\ 0, & n \in \mathbb{Z} \backslash Z_{+},\end{cases}
$$

and $\left\{a_{n}\right\}_{n \in \mathbb{Z}_{+}} \in l_{+}^{q},\left|a_{n}\right|=\left\|f_{n}\right\|_{X^{p, \lambda}(I)}, n \in \mathbb{Z}_{+} ;$moreover,

$$
\|f\|_{\mathscr{A} q ; p, \lambda(\mathbb{D})}=\|f\|_{\mathscr{A}_{\mathrm{loc}}^{q ; p, \lambda}(\mathbb{D})}=\|f\|_{\mathrm{c}_{\mathscr{A} q} ; p, \lambda(\mathbb{D})}=\left\|\left\{a_{n}\right\}_{n \in \mathbb{Z}_{+}}\right\|_{l_{+}^{q}}
$$

for the admissible range of the parameters $q, p, \lambda$ in each case.

\section{ESTIMATES OF THE NORMS $\left\|r^{n}\right\|_{L^{p, \lambda}(I)},\left\|r^{n}\right\|_{L_{\text {loc }}^{p, \lambda}(I)},\left\|r^{n}\right\|_{C_{L^{p, \lambda}(I)}}$}

Due to Remark 1, the behavior of $\left\|r^{n}\right\|_{X^{p, \lambda}(I)}$ as $n \rightarrow \infty$ plays an important role in our study.

Lemma 1. Let $1 \leq p<\infty, 0 \leq \lambda<1$. Then the following bilateral estimate:

$$
C_{1} n^{(\lambda-1) / p} \leq\left\|r^{n}\right\|_{L^{p, \lambda}(I)} \leq C_{2} n^{(\lambda-1) / p}, \quad n \rightarrow \infty,
$$

is valid, where $C_{1}, C_{2}$ are some positive constants which do not depend on $n$. 
Proof. Apply Hölder's inequality to the integral $(h>0, r \pm h \in I)$ :

$$
\begin{aligned}
\frac{1}{(2 h)^{\lambda}} \int_{r-h}^{r+h} t^{n p+1} 2 d t & \leq 2\left(\int_{r-h}^{r+h} t^{(n p+1)(1 /(1-\lambda))} d t\right)^{1-\lambda} \leq 2\left(\int_{I} t^{(n p+1)(1 /(1-\lambda))} d t\right)^{1-\lambda} \\
& =2\left((n p+1) \frac{1}{1-\lambda}+1\right)^{\lambda-1} .
\end{aligned}
$$

Hence, by definition,

$$
\left\|r^{n}\right\|_{L^{p, \lambda}(I)}=\sup _{r, r \pm h \in I, h>0}\left(\frac{1}{(2 h)^{\lambda}} \int_{r-h}^{r+h} t^{n p+1} 2 d t\right)^{1 / p} \leq 2^{1 / p}\left((n p+1) \frac{1}{1-\lambda}+1\right)^{(\lambda-1) / p} .
$$

Now we prove a similar estimate from below. Denote

$$
\begin{aligned}
E_{n} & =\left\{h \in I: 1-\left(\frac{1}{2}\right)^{1 /(n p+1)}<2 h<1-\left(\frac{1}{4}\right)^{1 /(n p+1)}\right\} \\
& =\left\{h \in I: \frac{1}{4}<(1-2 h)^{n p+1}<\frac{1}{2}\right\} .
\end{aligned}
$$

We have

$$
\begin{aligned}
\left\|r^{n}\right\|_{L^{p, \lambda}(I)}^{p} & \geq \sup _{r+h=1,0<h<1 / 2} \frac{1}{(2 h)^{\lambda}} \int_{1-2 h}^{1} t^{n p+1} 2 d t \\
& =2 \sup _{0<h<1 / 2} \frac{1}{(2 h)^{\lambda}}\left(\int_{1-2 h}^{1} t^{n p+1} d t\right)^{\lambda}\left(\int_{1-2 h}^{1} t^{n p+1} d t\right)^{1-\lambda} \\
& \geq 2 \sup _{h \in E_{n}}(1-2 h)^{(n p+1) \lambda}\left(\frac{1-(1-2 h)^{n p+2}}{n p+2}\right)^{1-\lambda} \\
& \geq 24^{-\lambda}\left(1-\frac{1}{2} \frac{1}{2^{1 /(n p+1)}}\right)^{1-\lambda}(n p+2)^{\lambda-1} \geq 2^{-\lambda}(n p+2)^{\lambda-1} .
\end{aligned}
$$

This concludes the proof.

Lemma 2. The following asymptotics are valid:

(1) $\left\|r^{n}\right\|_{L_{\text {loc }}^{p, \lambda}(I)} \sim n^{(\lambda-1) / p}, n \rightarrow \infty, 1 \leq p<\infty, 0 \leq \lambda<1$,

(2) $\left\|r^{n}\right\|_{C_{L^{p, \lambda}(I)}} \sim n^{(-\lambda-1) / p}, n \rightarrow \infty, 1<p<\infty, 0 \leq \lambda<p-1$.

Proof. The proof of this result is a matter of calculation and then taking supremum in $h \in I$. For instance,

$$
\left\|r^{n}\right\|_{C_{L^{p, \lambda}(I)}}^{p}=\sup _{h \in I} 2 h^{\lambda} \frac{(1-h)^{n p+2}}{n p+2}=\frac{2}{n p+2}\left(\frac{\lambda}{n p+2+\lambda}\right)^{\lambda} .
$$

Remark 2. It follows from Lemmas 1,2 that the spaces $\mathscr{A}^{q ; p, \lambda}(\mathbb{D})$ and $\mathscr{A}_{\text {loc }}^{q ; p, \lambda}(\mathbb{D})$ coincide up to the norm equivalence, $1 \leq q<\infty, 1 \leq p<\infty, 0 \leq \lambda<1$. However, the spaces $\mathscr{L}^{q ; p, \lambda}(\mathbb{D})$ and $\mathscr{L}_{\text {loc }}^{q ; p, \lambda}(\mathbb{D})$ are different. 


\section{BOUNDEDNESS OF THE BERGMAN PROJECTION}

First, we provide the reader with some preliminary results.

Lemma $3([23])$. Given a function $f$ in $L^{1}(\mathbb{D})$ let $f_{n}=f_{n}(r), n \in \mathbb{Z}$, denote the Fourier coefficients of the function $f$. Then the Fourier coefficients of the function $B_{\mathbb{D}} f$ are

$$
\begin{aligned}
& \left(B_{\mathbb{D}} f\right)_{n}(r)=r^{n}(n+1) \int_{I} \tau^{n} f_{n}(\tau) 2 \tau d \tau, \quad n \in \mathbb{Z}_{+}, \\
& \left(B_{\mathbb{D}} f\right)_{n}(r)=0, \quad n \in \mathbb{Z} \backslash \mathbb{Z}_{+} .
\end{aligned}
$$

Proof. The proof is just a matter of direct calculations.

We will need estimates for $\left\|\left(B_{\mathbb{D}} f\right)_{n}\right\|_{X^{p, \lambda}(I)}, n \in \mathbb{Z}_{+}$. The key result here is the following lemma.

Lemma 4. Let $g \in X^{p, \lambda}(I)$, where $X^{p, \lambda}(I)$ is any of the three spaces $L^{p, \lambda}(I), L_{\text {loc }}^{p, \lambda}(I)$, or ${ }^{\complement} L^{p, \lambda}(I)$. Then

$$
\left|\int_{I} \tau^{n} g(\tau) 2 \tau d \tau\right| \leq C\|g\|_{X^{p, \lambda}(I)} n^{-\lambda^{*} / p-1 / p^{\prime}}, \quad n \rightarrow \infty .
$$

where we denote $\lambda^{*}=\lambda$ in the case of Morrey $L^{p, \lambda}(I)$ and local Morrey $L_{\text {loc }}^{p, \lambda}(I)$ spaces and $\lambda^{*}=-\lambda$ in the case of complementary Morrey space ${ }^{\complement} L^{p, \lambda}(I)$, and the constant $C$ does not depend on either $f$ or $n$.

Proof. Passing to the dyadic decomposition over the intervals $I_{k}=\left(1-2^{-k}, 1-2^{-k-1}\right), k \in \mathbb{Z}_{+}$, we have

$$
\int_{I} \tau^{n} g(\tau) 2 \tau d \tau=2 \sum_{k \in \mathbb{Z}_{+}} \int_{I_{k}} \tau^{n+1} g(\tau) d \tau
$$

Let, as usual, $1 / p+1 / p^{\prime}=1$. Below we will proceed with the case $p>1$. Using Hölder's inequality we obtain

$$
\left|\int_{I_{k}} \tau^{n+1} g(\tau) d \tau\right| \leq\left(\int_{I_{k}} \tau^{(n+1) p^{\prime}}(1-\tau)^{\lambda^{*} p^{\prime} / p} d \tau\right)^{1 / p^{\prime}}\left(\int_{I_{k}}(1-\tau)^{-\lambda^{*}}|g(\tau)|^{p} d \tau\right)^{1 / p} .
$$

For each $k \in \mathbb{Z}_{+}$in the case of Morrey $L^{p, \lambda}(I)$ space, we have

$$
\left(\int_{I_{k}}(1-\tau)^{-\lambda}|g(\tau)|^{p} d \tau\right)^{1 / p} \leq\left(2^{(k+1) \lambda} \int_{I_{k}}|g(\tau)|^{p} d \tau\right)^{1 / p} \leq\|g\|_{L^{p, \lambda}(I)} .
$$

An analogous estimate holds in the case of local Morrey space $L_{\mathrm{loc}}^{p, \lambda}(I)$, but with the additional constant multiplier $2^{\lambda / p}$ in the right-hand side. Simultaneously, in the case of complementary Morrey space $X^{p, \lambda}(I)={ }^{\complement} L^{p, \lambda}(I)$, we have

$$
\left(\int_{I_{k}}(1-\tau)^{\lambda}|g(\tau)|^{p} d \tau\right)^{1 / p} \leq\left(2^{-k \lambda} \int_{I_{k}}|g(\tau)|^{p} d \tau\right)^{1 / p} \leq 2^{\lambda / p}\|g\|_{C_{L^{p, \lambda}}(I)} .
$$

Further, if we denote

$$
J_{n, \lambda^{*}, p^{\prime}}(k)=\left(\int_{I_{k}} \tau^{(n+1) p^{\prime}}(1-\tau)^{\lambda^{*} p^{\prime} / p} d \tau\right)^{1 / p^{\prime}},
$$

then direct estimates give

$$
\begin{aligned}
J_{n, \lambda, p^{\prime}}(k) & \leq 2^{\lambda / p}\left(1-2^{-k-1}\right)^{(n+1)}\left(2^{-k-1}\right)^{\lambda / p+1 / p^{\prime}}, \\
J_{n,-\lambda, p^{\prime}}(k) & \leq\left(1-2^{-k-1}\right)^{(n+1)}\left(2^{-k-1}\right)^{-\lambda / p+1 / p^{\prime}} .
\end{aligned}
$$


Hence if again $X^{p, \lambda}(I)$ is any of the three spaces $L^{p, \lambda}(I), L_{\text {loc }}^{p, \lambda}(I)$, or ${ }^{\complement} L^{p, \lambda}(I)$, then

$$
\left|\int_{I} \tau^{n} g(\tau) 2 \tau d \tau\right| \leq 2^{1+\lambda / p} \delta_{p, \lambda}\|g\|_{X^{p, \lambda}(I)} \sum_{k \in \mathbb{Z}_{+}}\left(1-2^{-k-1}\right)^{(n+1)}\left(2^{-k-1}\right)^{\lambda^{*} / p+1 / p^{\prime}},
$$

where $\delta_{p, \lambda}=1$, if either $X^{p, \lambda}(I)=L^{p, \lambda}(I)$ or $X^{p, \lambda}(I)={ }^{\complement} L^{p, \lambda}(I)$ and $\delta_{p, \lambda}=2^{\lambda / p}$ in the case of local Morrey space $L_{\text {loc }}^{p, \lambda}(I)$. Note that $0<\lambda^{*} / p+1 / p^{\prime}<1$. We have

$$
\begin{gathered}
\sum_{k \in \mathbb{Z}_{+}}\left(1-2^{-k-1}\right)^{(n+1)}\left(2^{-k-1}\right)^{\lambda^{*} / p+1 / p^{\prime}} \leq 2 \sum_{k \in \mathbb{Z}_{+}} \int_{I_{k+1}} \tau^{n+1}(1-\tau)^{\lambda^{*} / p+1 / p^{\prime}-1} d \tau \\
=2 \int_{1 / 2}^{1} \tau^{n+1}(1-\tau)^{\lambda^{*} / p+1 / p^{\prime}-1} d \tau \leq 2 \mathrm{~B}\left(n+2, \frac{\lambda^{*}}{p}+\frac{1}{p^{\prime}}\right),
\end{gathered}
$$

where $\mathrm{B}(x, y)$ is the Euler's beta function. According to the known asymptotic of the Euler's beta function:

$$
\mathrm{B}\left(n+2, \frac{\lambda^{*}}{p}+\frac{1}{p^{\prime}}\right) \sim(n+2)^{-\lambda^{*} / p-1 / p^{\prime}}, \quad n \rightarrow \infty .
$$

Gathering the above estimates, we end up with (5.2) for the case $p>1$.

Let now $p=1$. In that case we need to prove the statement for Morrey $L^{p, \lambda}(I)$ and local Morrey $L_{\text {loc }}^{p, \lambda}(I)$ spaces. The proof is similar to the proof for considered above case $p>1$ : one needs to use the $L^{\infty}\left(I_{k}\right)$-norm instead of the corresponding integral norm.

Lemma 5. For $f \in L^{1}(\mathbb{D})$ and $n \in \mathbb{Z}_{+}$, one has

(1) $\left\|\left(B_{\mathbb{D}} f\right)_{n}\right\|_{L^{p, \lambda}(I)} \leq C\left\|f_{n}\right\|_{L^{p, \lambda}(I)}, 1 \leq p \leq \infty, 0 \leq \lambda<1$,

(2) $\left\|\left(B_{\mathbb{D}} f\right)_{n}\right\|_{L_{\text {loc }}^{p, \lambda}(I)} \leq C\left\|f_{n}\right\|_{L_{\text {loc }}^{p, \lambda}(I)}, 1 \leq p \leq \infty, 0 \leq \lambda<1$,

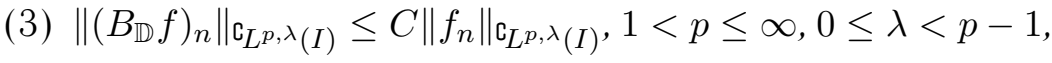

where the constant $C$ does not depend on either $f$ or $n$.

Proof. Let us show the validity of the first statement. The proofs for the two remaining ones are similar. Using (5.1), we get the following expression for the $L^{p, \lambda}(I)$-norm for $\left(B_{\mathbb{D}} f\right)_{n}, n \in \mathbb{Z}_{+}$:

$$
\left\|\left(B_{\mathbb{D}} f\right)_{n}\right\|_{L^{p, \lambda}(I)}=\left\|r^{n}\right\|_{L^{p, \lambda}(I)}(n+1)\left|\int_{I} \tau^{n} f_{n}(\tau) 2 \tau d \tau\right| .
$$

It remains to use Lemmas 1,2 , and 4 .

Now we are in position to prove the boundedness of the Bergman projection. The Bergman projection $B_{\mathbb{D}}$ is understood as a continuous extension from a dense subset (see below).

Theorem 6. The operator $B_{\mathbb{D}}$ is bounded as a projection

(1) from $\mathscr{L}^{q ; p, \lambda}(\mathbb{D})$ onto $\mathscr{A}^{q ; p, \lambda}(\mathbb{D}), 1 \leq q<\infty, 1 \leq p<\infty, 0 \leq \lambda<1$;

(2) from $\mathscr{L}_{\text {loc }}^{q ; p, \lambda}(\mathbb{D})$ onto $\mathscr{A}_{\text {loc }}^{q ; p, \lambda}(\mathbb{D}), 1 \leq q<\infty, 1 \leq p<\infty, 0 \leq \lambda<1$;

(3) $\operatorname{from}^{\complement} \mathscr{L}^{q ; p, \lambda}(\mathbb{D})$ onto $\complement_{\mathscr{A}}^{q ; p, \lambda}(\mathbb{D}), 1 \leq q<\infty, 1<p<\infty, 0 \leq \lambda<p-1$. 
Proof. We will prove the first statement. The other cases can be treated similarly. Let $\mathscr{L}_{0}^{p, \lambda}(\mathbb{D})$ denote the set of functions of the form

$$
f(z)=f\left(r, e^{i \alpha}\right)=\sum_{n=-N}^{N} f_{n}(r) e^{i n \alpha}, \quad f_{n} \in L^{p, \lambda}(I),
$$

where $N \in \mathbb{Z}_{+}$is arbitrary. It is evident that $\mathscr{L}_{0}^{p, \lambda}(\mathbb{D})$ is dense in $\mathscr{L}^{q ; p, \lambda}(\mathbb{D}), 1 \leq q<\infty, 1 \leq p<\infty$. The Bergman projection $B_{\mathbb{D}}$ is well defined on functions of such type since $\mathscr{L}_{0}^{p, \lambda}(\mathbb{D}) \subset L^{1}(\mathbb{D})$. Lemma 5 is applicable for $f \in \mathscr{L}_{0}^{p, \lambda}(\mathbb{D})$ and we obtain

$$
\left\|B_{\mathbb{D}} f\right\|_{\mathscr{L} q ; p, \lambda(\mathbb{D})}^{q}=\sum_{-N}^{N}\left\|\left(B_{\mathbb{D}} f\right)_{n}\right\|_{L^{p, \lambda}(I)}^{q} \leq C^{q} \sum_{-N}^{N}\left\|f_{n}\right\|_{L^{p, \lambda}(I)}^{q}=C^{q}\|f\|_{\mathscr{L}^{q ; p, \lambda}(\mathbb{D})}^{q},
$$

where the constant $C$ comes from Lemma 4 and does not depend on $f$. Making use of Banach-Steinhaus theorem, we conclude the proof.

Corollary 7. Under the conditions on the parameters $q, p, \lambda$ stated in Theorem 6 , the spaces $\mathscr{A}^{q ; p, \lambda}(\mathbb{D}), \mathscr{A}_{\mathrm{loc}}^{q ; p, \lambda}(\mathbb{D})$, and $\complement_{\mathscr{A}^{q ; p, \lambda}}(\mathbb{D})$ are closed subspaces of $\mathscr{L}^{q ; p, \lambda}(\mathbb{D}), \mathscr{L}_{\mathrm{loc}}^{q ; p, \lambda}(\mathbb{D})$, and $\complement_{\mathscr{L}^{q ; p, \lambda}}(\mathbb{D})$, respectively.

\section{FURTHER PROPERTIES OF $\mathscr{A}^{q ; p, \lambda}(\mathbb{D})$ AND ${ }^{\complement} \mathscr{A}^{q ; p, \lambda}(\mathbb{D})$}

In view of Remark 2, here we will proceed by exploring further properties of Bergman-Morrey $\mathscr{A}^{q ; p, \lambda}(\mathbb{D})$ space and Bergman-Morrey ${ }^{\complement} \mathscr{A}^{q ; p, \lambda}(\mathbb{D})$ space of complementary type.

Let us introduce the weighted Lebesgue space $L_{(1-r)^{\gamma}}^{p}(I)$ as the space of measurable functions $g$ on $I$ with the norm

$$
\|g\|_{L_{(1-r)^{\gamma}}^{p}(I)}=\left(\int_{I}|g(r)|^{p}(1-r)^{\gamma} 2 r d r\right)^{1 / p}, \quad \gamma \in \mathbb{R}, \quad 1 \leq p<\infty .
$$

Theorem 8. Let $f(z)=\sum_{n \in \mathbb{Z}_{+}} c_{n} z^{n}, z \in \mathbb{D}$. Then

(1) the norm $\|f\|_{\mathscr{A} q ; p, \lambda(\mathbb{D})}$ of a function $f \in \mathscr{A}^{q ; p, \lambda}(\mathbb{D}), 1 \leq q<\infty, 1 \leq p<\infty, 0 \leq \lambda<1$, is equivalent to each one of the following two expressions:

$$
\left(\sum_{n \in \mathbb{Z}_{+}}(n+1)^{q(\lambda-1) / p}\left|c_{n}\right|^{q}\right)^{1 / q}, \quad\left(\sum_{n \in \mathbb{Z}_{+}}\left\|f_{n}\right\|_{L_{(1-r)-\lambda}^{p}}^{q}(I)\right)^{1 / q}
$$

(2) the norm $\|f\|_{\mathscr{A}^{q} ; p, \lambda(\mathbb{D})}$ of a function $f \in{ }^{\complement} \mathscr{A}^{q ; p, \lambda}(\mathbb{D}), 1 \leq q<\infty, 1<p<\infty, 0 \leq \lambda<p-1$, is equivalent to each one of the following two expressions:

$$
\left(\sum_{n \in \mathbb{Z}_{+}}(n+1)^{q(-\lambda-1) / p}\left|c_{n}\right|^{q}\right)^{1 / q}, \quad\left(\sum_{n \in \mathbb{Z}_{+}}\left\|f_{n}\right\|_{L_{(1-r) \lambda}^{p}}^{q}(I)\right)^{1 / q} .
$$

Proof. The proof follows from the definitions of the corresponding spaces in view of Remark 1, Lemmas 1 and 2, and the observation that

$$
\left\|r^{n}\right\|_{L_{(1-r) \mp \lambda}^{p}(I)}^{p}=2 \mathrm{~B}(n p+2,1 \mp \lambda) \sim 2(n p+2)^{-(1 \mp \lambda)}, \quad n \rightarrow \infty .
$$

Corollary 9. Let $1 \leq q<2$. The Hardy-Littlewood theorem (see [6, p. 76]) implies the continuous embeddings: 
(1) $H^{q}(\mathbb{D}) \hookrightarrow \mathscr{A}^{q ; p, \lambda}(\mathbb{D})$ if $q(1+(1-\lambda) / p) \geq 2,1 \leq p<\infty, 0 \leq \lambda<1$;

(2) $H^{q}(\mathbb{D}) \hookrightarrow^{\complement} \mathscr{A}^{q ; p, \lambda}(\mathbb{D})$ if $q(1+(1+\lambda) / p) \geq 2,1<p<\infty, 0 \leq \lambda<p-1$.

Let us define the space $\mathscr{A}_{\gamma}^{q ; p}(\mathbb{D}), \gamma \in \mathbb{R}, 1 \leq p<\infty$, as in the definition of Bergman-Morrey-type spaces, where, instead of the Morrey-type norms, we use the weighted Lebesgue norm (6.1), as suggested by Theorem 8 .

Theorem 10. The following relations:

(1) $\mathscr{A}^{q ; p, \lambda}(\mathbb{D})=\mathscr{A}_{\lambda}^{q ; p}(\mathbb{D}), 1 \leq q<\infty, 1 \leq p<\infty, 0 \leq \lambda<1$;

(2) ${ }^{\complement} \mathscr{A}^{q ; p, \lambda}(\mathbb{D})=\mathscr{A}_{-\lambda}^{q ; p}(\mathbb{D}), 1 \leq q<\infty, 1<p<\infty, 0 \leq \lambda<p-1$,

are valid up to norm equivalence.

Proof. The proof follows from Theorem 8.

As another corollary of Theorem 8 we see that for the case $q=2$ the spaces under consideration are represented as Hadamard-type fractional derivatives of functions in Hardy space $H^{2}(\mathbb{D})$. For fractional integro-differentiation we refer the reader to [26]. The operation with multiplication of coefficients of

$$
f(z)=\sum_{n \in \mathbb{Z}_{+}} c_{n} z^{n}
$$

by $(n+1)^{\alpha}, \alpha>0$, is the fractional differentiation of Hadamard. We will denote this operator by $D^{\alpha}$ and for the corresponding fractional integration operator we use $I^{\alpha}$. They are well defined on the whole set of all functions $f$ analytic in the disc $\mathbb{D}$, preserve this set, and $D^{\alpha} I^{\alpha} f=I^{\alpha} D^{\alpha} f=f$ for all such $f$ (see [26] and [27]).

Theorem 11. Let $q=2$. Then the following relations:

(1) $\mathscr{A}^{2 ; p, \lambda}(\mathbb{D})=D^{(1-\lambda) / p}\left(H^{2}(\mathbb{D})\right), 1 \leq p<\infty, 0 \leq \lambda<1$;

(2) ${ }^{\complement} \mathscr{A}^{2 ; p, \lambda}(\mathbb{D})=D^{(1+\lambda) / p}\left(H^{2}(\mathbb{D})\right), 1<p<\infty, 0 \leq \lambda<p-1$,

hold up to norm equivalence.

Proof. The proof follows from Theorem 8 due to the known fact that

$$
f(z)=\sum_{n \in \mathbb{Z}_{+}} c_{n} z^{n} \in H^{2}(\mathbb{D}) \Longleftrightarrow\|f\|_{H^{2}(\mathbb{D})}^{2}=\sum_{n \in \mathbb{Z}_{+}}\left|c_{n}\right|^{2}<\infty .
$$

Following [27], we introduce the mixed-norm space $H(s, t, \gamma), s>0, t>0, \gamma>0$, of measurable on $\mathbb{D}$ functions with the norm

$$
\begin{aligned}
\|f\|_{H(s, t, \gamma)} & =\left\{\int_{I}(1-r)^{t \gamma-1} \mathscr{M}_{s}^{t}(f ; r) d r\right\}^{1 / t}, \quad 0<t<\infty, \\
\|f\|_{H(s, \infty, \gamma)} & =\sup _{I}\left\{(1-r)^{\gamma} \mathscr{M}_{s}(f ; r)\right\}, \quad t=\infty .
\end{aligned}
$$

Information about such spaces, including embedding theorems, may be found in [28].

The following result shows that at least for $q \leq 2$ the functions in our spaces are regular in the sense that they have finite Lebesgue-type mixed norm.

Theorem 12. Let $1<p<\infty, 1 \leq q \leq 2$. Then the following continuous embeddings hold: 
(1) $\mathscr{A}^{q ; p, \lambda}(\mathbb{D}) \hookrightarrow H(s, t, 1 / 2-1 / s+(1-\lambda) / p) \hookrightarrow L^{1}(\mathbb{D}), \quad 0 \leq \lambda<1$, if $(1-\lambda) / p<1 / 2+1 / s$, $2<s \leq \infty, 2 \leq t \leq \infty$

(2) $\complement_{\mathscr{A}^{q ; p, \lambda}}(\mathbb{D}) \hookrightarrow H(s, t, 1 / 2-1 / s+(1+\lambda) / p) \hookrightarrow L^{1}(\mathbb{D}), 0 \leq \lambda<p-1$, if $(1+\lambda) / p<1 / 2+1 / s$, $2<s \leq \infty, 2 \leq t \leq \infty$.

Proof. In view of $\mathscr{A}^{q ; p, \lambda}(\mathbb{D}) \hookrightarrow \mathscr{A}^{2 ; p, \lambda}(\mathbb{D})$ and $\complement_{\mathscr{A}}^{q ; p, \lambda}(\mathbb{D}) \hookrightarrow^{\complement} \mathscr{A}^{2 ; p, \lambda}(\mathbb{D})$ for $1 \leq q \leq 2$, it suffices to prove the theorem for $q=2$.

According to Flett's result (see [27, Theorem B and Theorem 6]), for the given function $f \in H^{2}(\mathbb{D})$, one has the estimate $\left\|D^{\alpha} f\right\|_{H(s, t, 1 / 2-1 / s+\alpha)} \leq C\|f\|_{H^{2}(\mathbb{D})}$, provided $2<s \leq \infty, 2 \leq t \leq \infty, \alpha>0$, where $C$ does not depend on $f$. This estimate, along with Theorem 11, implies the embeddings

$$
\mathscr{A}^{2 ; p, \lambda}(\mathbb{D}) \hookrightarrow H\left(s, t, \frac{1}{2}-\frac{1}{s}+\frac{1-\lambda}{p}\right), \quad \complement_{\mathscr{A}^{2 ; p, \lambda}}(\mathbb{D}) \hookrightarrow H\left(s, t, \frac{1}{2}-\frac{1}{s}+\frac{1+\lambda}{p}\right) .
$$

To prove $H(s, t, 1 / 2-1 / s+(1 \mp \lambda) / p) \hookrightarrow L^{1}(\mathbb{D})$, one must repeatedly use Hölder's inequality under the following condition on the parameter $s$ :

$$
\frac{1 \mp \lambda}{p}<\frac{1}{2}+\frac{1}{s}, \quad 2<s \leq \infty
$$

\section{ACKNOWLEDGMENTS}

S. G. Samko's work was supported by the Russian Foundation for Basic Research under grant 15-01-02732.

\section{REFERENCES}

1. S. Bergman, "Über die Kernfunktion eines Bereiches und ihr Verhalten am Rande. I," J. Reine Angew. Math. 169, 1-42(1932).

2. M. M. Dzherbashyan, "On canonical representation of functions meromorphic in the unit disc," Dokl. Akad. Nauk ArmSSR 3 (1), 3-9 (1945).

3. M. M. Dzherbashyan, "On the representability problem for analytic functions," Soobsh. Inst. Mat. i Mekh. Akad. Nauk ArmSSR, No. 2, 3-55 (1948).

4. S. Bergman, The Kernel Function and Conformal Mapping, in Math. Surveys Monogr. (Amer. Math. Soc., Providence, RI, 1970), Vol. 5.

5. A. E. Djrbashian and F. A. Shamoian, Topics in the Theory of $A_{\alpha}^{p}$ Spaces, in Teubner-Texte zur Mathematik (BSB B. G. Teubner Verlagsgesellschaft, Leipzig, 1988), Vol. 105.

6. P. Duren and A. Schuster, Bergman Spaces, in Math. Surveys Monogr. (Amer. Math. Soc., Providence, RI, 2004), Vol. 100.

7. H. Hedenmalm, B. Korenblum, and K. Zhu, Theory of Bergman Spaces, in Graduate Texts in Math. (Springer-Verlag, New York, 2000), Vol. 199.

8. K. Zhu, Operator Theory in Function Spaces, in Math. Surveys Monogr. (Amer. Math. Soc., Providence, RI, 2007), Vol. 138.

9. K. Zhu, Spaces of Holomorphic Functions in the Unit Ball, in Graduate Texts in Math. (Springer-Verlag, New York, 2004), Vol. 226.

10. N. L. Vasilevski, Commutative Algebras of Toeplitz Operators on the Bergman Space, in Operator Theory: Adv. Appl. (Birkhäuser Verlag, Basel, 2008), Vol. 185.

11. D. Gu, "Bergman projections and duality in weighted mixed-norm spaces of analytic functions," Michigan Math. J. 39 (1), 71-84 (1992).

12. M. Jevtić, "Bounded projections and duality in mixed-norm spaces of analytic functions," Complex Variables Theory Appl. 8 (3-4), 293-301 (1987).

13. S. Gadbois, "Mixed norm generalizations of Bergman spaces and duality," Proc. Amer. Math. Soc. 104 (4), $1171-1180(1988)$.

14. Y. Liu, "Boundedness of the Bergman-type operators on mixed-norm space," Proc. Amer. Math. Soc. 130 (8), 2363-2367 (2002).

15. S. Li, "A class of integral operators on mixed-norm spaces in the unit ball," Czechoslovak Math. J. 57 (132) (3), 1013-1023 (2007).

16. A. Kufner, O. John, and S. Fučik, Function Spaces (Noordhoff Int. Publ., Leyden, 1977). 
17. M. Giaquinta, Multiple Integrals in the Calculus of Variations and Nonlinear Elliptic Systems, in Ann. of Math. Stud. (Princenton Univ. Press, Princenton, NJ, 1977), Vol. 105.

18. N. Samko, S. Samko, and H. Rafeiro, "Morrey-Campanato spaces: an overview," in Operator Theory, Pseudo-Differential Equations, and Mathematical Physics, Oper. Theory Adv. Appl. (Springer, Basel, 2013), Vol. 228, pp. 293-323.

19. V. I. Burenkov, "Recent progress in studying the boundedness of classical operators of real analysis in general Morrey-type spaces. I," Eurasian Math. J. 3 (3), 11-32 (2012).

20. V. I. Burenkov, "Recent progress in studying the boundedness of classical operators of real analysis in general Morrey-type spaces. II," Eurasian Math. J. 4 (1), 21-45 (2013).

21. V. Guliev, J. Hasanov, and S. Samko, "Boundedness of the maximal, potential and singular operators in the generalized variable exponent Morrey spaces," Math. Scand. 107 (2), 285-304 (2010).

22. V. S. Guliev, J. J. Hasanov, and S. G. Samko, "Boundedness of the maximal, potential and singular integral operators in the generalized variable exponent Morrey-type spaces," J. Math. Sci. 170 (4), 423-443 (2010).

23. A. Karapetyants and S. Samko, "Mixed norm variable exponent Bergman space on the unit disc," Complex Var. Elliptic Equ. (2016) (in press).

24. Z. Wu and C. Xie, “Q spaces and Morrey spaces,” J. Funct. Anal. 201 (1), 282-297 (2003).

25. J. Liu and Z. Lou, "Properties of analytic Morrey spaces and applications," Math. Nachr. 288 (14-15), 1673-1693 (2015).

26. S. Samko, A. Kilbas, and O. I. Marichev, Fractional Integrals and Derivatives: Theory and Applications (Gordon and Breach Sci. Publ., Yverdon, 1993).

27. T. M. Flett, "The dual of an inequality of Hardy and Littlewood and some related inequalities," J. Math. Anal. and Appl. 38 (3), 746-765 (1972).

28. I. Arévalo, "A characterization of the inclusions between mixed-norm spaces," J. Math. Anal. Appl. 429 (5), 942-955 (2015). 